\title{
PRESCRIPCIÓN DE EJERCICIO TERAPÉUTICO EN FISIOTERAPIA. LAS BASES ELEMENTALES DE LA IDENTIDAD PROFESIONAL
}

\author{
Roy La Touche, $\mathrm{PhD}^{1,2,3}$ \\ 1. Departamento de Fisioterapia, Facultad de Ciencias de la Salud. Centro Superior de Estudios Universitarios La Salle, Universidad Autónoma de Madrid, \\ Madrid, España. \\ 2. Motion in Brains Research Group, Instituto de Neurociencias y Ciencias del Movimiento (INCIMOV), Centro Superior de Estudios Universitarios La Salle, \\ Universidad Autónoma de Madrid, Madrid, España. \\ 3. Instituto de Neurociencia y Dolor Craneofacial (INDCRAN), Madrid, España.
}

\section{Correspondencia:}

Roy La Touche, PT, PhD Departamento de Fisioterapia. CSEU La Salle. Universidad Autónoma of Madrid. Calle La Salle, $\mathrm{n}^{\circ} 10,28023$ Madrid, España Teléfono: (+34) 917401980

Fax: (+34) 913571730

E-mail: Roylatouche@yahoo.es

DOI:

https://doi.org/10.37382/jomts.v2i1.20

Licensed under: CC BY-NC-SA 4.0

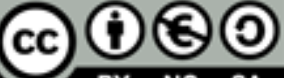
Access to legal code
Históricamente la concepción profesional de la fisioterapia se asienta a través de la utilización de la terapia manual y ejercicio como medios terapéuticos principales para recuperar a pacientes con diversos trastornos (Hansson \& Ottosson, 2015; Shaik \& Maniyat Shemjaz, 2014). Los términos movimiento y ejercicio están directamente relacionados con la génesis y evolución de la fisioterapia. Algunos autores sugieren que la construcción central del cuerpo del conocimiento de la fisioterapia es el sistema de movimiento humano (Coffin-Zadai, 2007; Sahrmann, 2014; Voight \& Hoogenboom, 2017), este sistema se define como "la integración de sistemas corporales que generan y mantienen el movimiento en todos los niveles de la función corporal y está influenciado por factores sociales, ambientales y personales"(American Physical Therapy Association, 2015).

La identidad profesional del fisioterapeuta ha sido motivo de múltiples reflexiones a lo largo del tiempo. Sahrmann planteó la necesidad de establecer la identidad profesional de los fisioterapeutas como especialistas en el sistema del movimiento humano (Sahrmann, 2014). Por otra parte, Cofffin-Zadai sugirió en una declaración de intenciones de perfil teórico, una reflexión que a día de hoy siguen estando vigente; "Necesitamos reconocimiento profesional y público de quiénes somos y qué hacemos. Los fisioterapeutas deben ser dueños del sistema de movimiento humano y su manejo desde la ciencia hasta la práctica" (Coffin-Zadai, 2007). La Asociación Americana de Fisioterapia (APTA), respecto a la identidad profesional declara que "La profesión de fisioterapia definirá y promoverá el sistema de movimiento como la base para optimizar el movimiento para mejorar la salud de la sociedad", además añade que "el sistema de movimiento es el núcleo de la práctica, educación e investigación del fisioterapeuta"(American Physical Therapy Association, 2015).

Las necesidades sociales, científicas y sanitarias que demanda la actualidad entorno a la rehabilitación del paciente, exigen que el fisioterapeuta se desenvuelva en un modelo en donde el razonamiento clínico, el diagnóstico, el pronóstico y el planteamiento terapéutico se establezca a través del estudio y análisis del sistema del movimiento humano. Introducir modelo de este tipo reduciría la dependencia del modelo fisiopatológico que es el predominante. Un modelo de fisioterapia basado en el comportamiento motor por definición es multidimensional, siendo la dimensión de discapacidad funcional principal elemento a considerar a nivel de diagnóstico y de toma de decisiones. Las dimensiones circundantes serían la cognitiva-conductual, la afectiva-motivacional y la sensorial. 
Este modelo o marco de trabajo se adapta adecuadamente a los abordajes de fisioterapia de los trastornos cardiovasculares, pulmonares, musculoesqueléticos y neuromusculoesqueléticos. La dimensión de discapacidad funcional como elemento principal de este modelo (fisioterapia basado en el comportamiento motor) incluye tres factores que desgranan esta dimensión según la Clasificación Internacional del Funcionamiento: la deficiencia, la limitación en la actividad y la restricción en la actividad (Fernández-López, Fernández-Fidalgo, \& Cieza, 2010).

Las deficiencias se refieren a los problemas en las funciones de estructuras corporales, las limitaciones en la actividad son dificultades que un individuo puede tener en el desempeño/realización de actividades y la restricción en la participación son problemas que el individuo puede experimentar al involucrarse en situaciones vitales (Figura 1), estos factores pueden verse influidos por las otras dimensiones (cognitivaconductual, la afectiva-motivacional, la sensorial) y otros factores interdependientes en el comportamiento motor como la adherencia, la alianza terapéutica, la calidad de vida y la fatiga (Figura 2).

Ajustar los modelos de diagnóstico y tratamiento basado en el sistema del movimiento puede incrementar la efectividad del ejercicio terapéutico que es el tratamiento que cuentan con mayor evidencia científica en la actualidad. A pesar de los efectos científicamente contrastados que tiene el ejercicio terapéutico, hay que tener en cuenta que hay problemas importantes para que su implementación sea mayoritaria o se realice de forma adecuada por parte de los fisioterapeutas. En relación con esto, Nyland sugiere que los fisioterapeutas deben de profesionalizar aún más su contribución y compromiso con la prescripción de ejercicio terapéutico si quieren aspirar a convertirse en los profesionales del sistema del movimiento (Nyland, 2015).

Cambios en la formación universitaria, en políticas de salud pública y en la participación y promoción de los colegios profesionales y sociedades científicas con respecto al ejercicio terapéutico son muy necesarias. Es importante consolidar las competencias asociadas a esta terapéutica e incrementar la identidad del fisioterapeuta alrededor del sistema del movimiento y la prescripción de ejercicio terapéutico como elemento esencial en la recuperación funcional del paciente. Las generaciones actuales, pero sobre todo las futuras, deben incorporar una filosofía en la que el movimiento sea el motor de decisión en la que rondan la mayoría de las decisiones e interpretaciones clínicas.

La comunidad sanitaria, científica y sobre todo la población general deben de identificar al fisioterapeuta como un profesional capacitado para establecer un diagnóstico funcional y una prescripción de ejercicio terapéutico para lograr una disminución de la discapacidad funcional efectiva y segura. La historia nos indica que hay un "ADN" fisioterápico asociado al movimiento, sin embargo parece que a nivel evolutivo algunos atributos tienden a difuminarse o degradarse conceptualmente hablando. Es el momento adecuado para reivindicar la figura del fisioterapeuta. Hay que recordar que en momentos claves de la historia, en donde se han producido catástrofes sanitarias o conflictos bélicos que han producido múltiples afectaciones, que a su vez han generado altos niveles de discapacidad, los fisioterapeutas estuvieron ahí recuperando a los pacientes a través del movimiento.

La fisioterapia es una profesión que incluye contenidos científicos de las ciencias aplicadas a la rehabilitación y casi en el mismo porcentaje de las ciencias del movimiento humano. Esto conlleva que la conceptualización de los procedimientos utilizados dentro de los marcos de actuación clínica tenga que adaptarse a las competencias y necesidades de la profesión. Un ejemplo de esto son los conceptos de actividad física y actividad física terapéutica, o los de ejercicio físico y ejercicio terapéutico, siendo los segundos los términos específicos a utilizar dentro del léxico fisioterápico. A continuación se detallan algunas definiciones que deben conocer los fisioterapeutas:

\section{- Actividad fisica}

Para cualquier movimiento corporal generado por la contracción de los músculos esqueléticos que aumenta el gasto de energía por encima de la tasa metabólica de reposo (Caspersen, Powell, \& Christenson, 1985; 
Garber et al., 2011). La actividad física incluye en general el ejercicio, los deportes y las actividades físicas realizadas como parte de la vida diaria, la recuperación de enfermedades y cualquier condición que perturbe su movimiento y actividad de la vida diaria (Kottke y Stillwell, 1982).

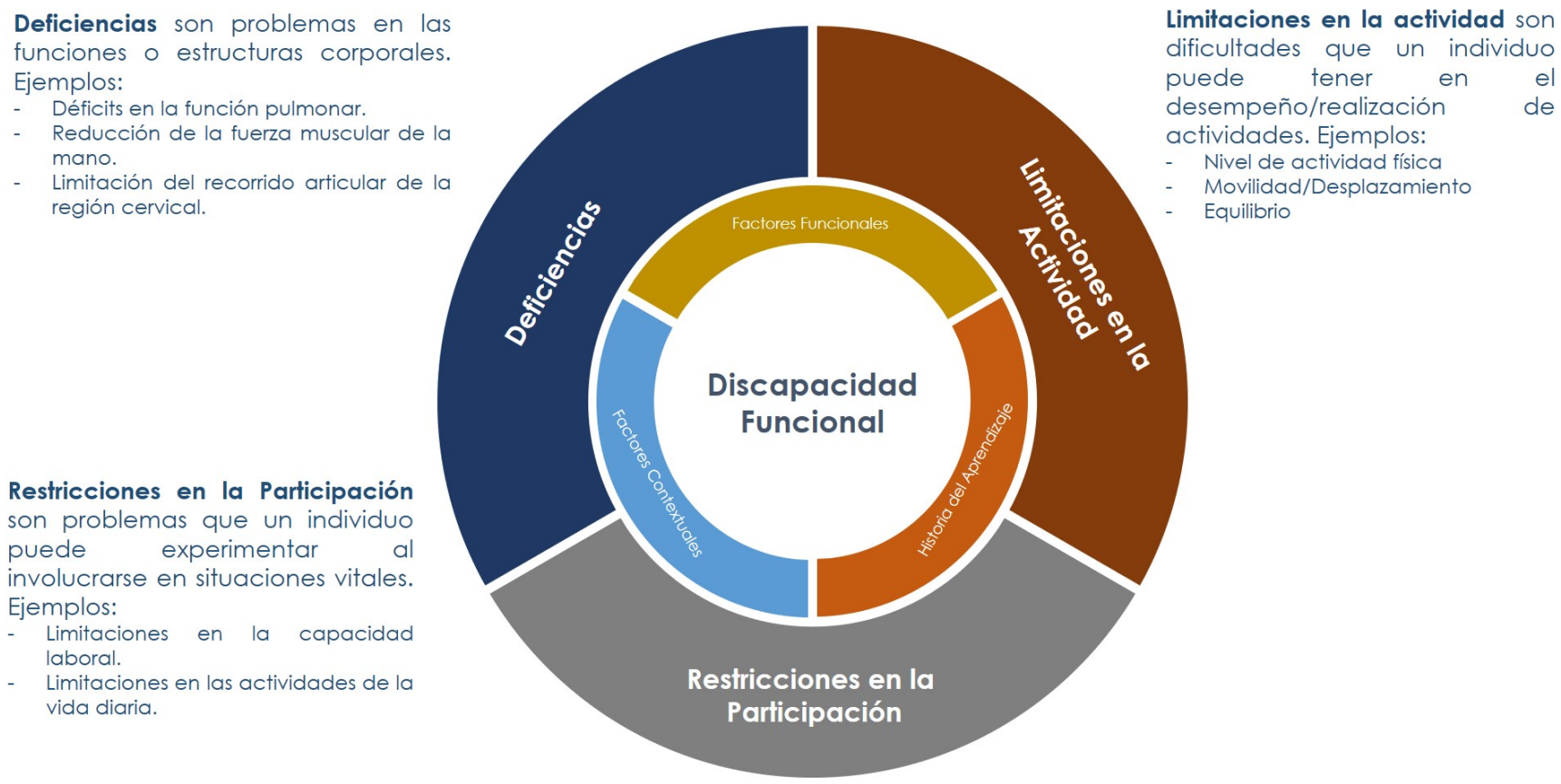

Figura 1. La figura representa la subdivisión de la dimensión motora (Discapacidad Funcional) del modelo de fisioterapia basado en el comportamiento motor. La subdivisión presenta tres ejes que se explican en la figura con sus respectivos ejemplos. Además, hay tres factores circundantes (factores contextuales, factores emocionales e historia del aprendizaje) que pueden influir sobre los tres ejes que conforman la discapacidad funcional. Los factores contextuales están relacionados con aspectos sociales, educacionales, con la edad, la profesión, etc. La historia de aprendizaje o experiencia previa está relacionada con lo aprendido anteriormente y como esto a su vez puede influir sobre el comportamiento. Los factores funcionales incluyen todos los mecanismos bilógicos que influyen sobre la ejecución, planificación y aprendizaje de un movimiento y que pueden condicionar las actividades físicas y la ejecución de ejercicios.

ocupación, el ocio y el transporte activo (Garber et al., 2011).

\section{- Actividad física terapéutica}

Son tareas funcionales dinámicas multiplanares que incluyen actividades de la vida diaria y se prescriben para recuperar o mejorar una función.

\section{- Ejercicio fisico}

Movimiento corporal planificado, estructurado y repetitivo realizado para mejorar o mantener uno o más componentes de la aptitud física (Caspersen et al., 1985; Garber et al., 2011).

\section{- Ejercicio Terapéutico}

Un plan de ejercicio físico o actividad física diseñadas y prescritas para facilitar a los pacientes la

\section{- Aptitud fisica}

Un conjunto de atributos medibles que están relacionados con la salud y las habilidades. Incluyen la aptitud cardiorrespiratoria, la fuerza/resistencia muscular, la composición corporal, la flexibilidad, el equilibrio, la agilidad, el tiempo de reacción y la potencia (Garber et al., 2011).

\section{- Deporte}

Es parte del espectro de actividad física $\mathrm{y}$ corresponde a cualquier práctica institucionalizada y organizada, controlada por reglas específicas (Thivel et al., 2018).

La actividad física terapéutica y el ejercicio terapéutico son dos modalidades de tratamiento que 
están incorporadas en la praxis de la fisioterapia donde la utilización de ambas debería realizarse mediante rigurosa prescripción de ejercicio terapéutico. utilizando el FITT-VP indicaría: 1) "frecuencia", explica los días concretos de la semana en donde se tiene que realizar el programa de ejercicio; 2) "intensidad, determina el porcentaje del RM o la tasa

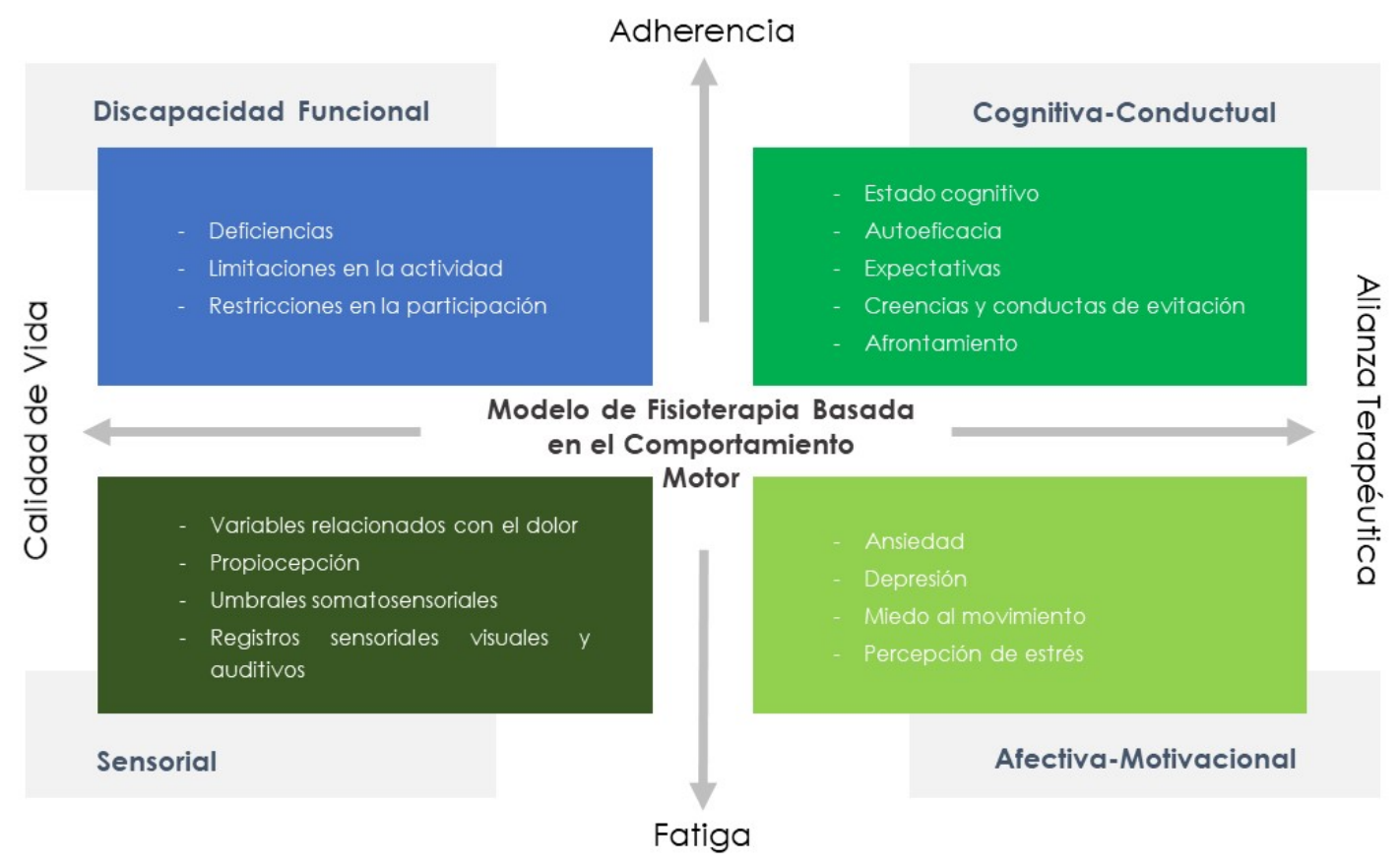

Figura 2. El modelo de Fisioterapia Basada en el Comportamiento Motor se encuentra dentro de un paradigma bioconductual, tiene 4 dimensiones (discapacidad funcional, sensorial, afectiva-motivacional y la cognitiva-conductual). La dimensión de discapacidad funcional representa el punto central en el que giran las otras dimensiones que influyen, interfieren y complementan los ejes relacionados con el comportamiento motor (deficiencias, limitaciones en la actividad y restricciones en la participación). Hay otros elementos multidimensionales e interdependientes como la fatiga, la calidad de vida, la alianza terapéutica y la adherencia que son transversales a las 4 dimensiones y además son fundamentales a nivel diagnóstico y en el incremento de la efectividad de los planteamientos terapéuticos basados en ejercicio.

Según La Touche, este es "un proceso sistemático en el cual se establece un planteamiento terapéutico basado en pruebas físicas y diseñado con el objetivo de recuperar, mejorar, prevenir y optimizar las funciones físicas mediante la utilización de ejercicios que presentan un énfasis rehabilitador" (La Touche, 2017).

El proceso sistemático, individualizado y específico en el que se establece la prescripción de ejercicio terapéutico se desarrolla a través de un principio conocido como FITT-VP (Zaleski et al., 2016), estas siglas hacen hincapié en los parámetros de prescripción a considerar desde el punto de vista práctico $(\mathrm{F}$, frecuencia; $\mathrm{I}$, intensidad; $\mathrm{T}$, tiempo; $\mathrm{T}$, tipo de ejercicio; V, volumen; P, progresión). Un ejemplo relacionado con el entrenamiento contra resistencia de esfuerzo percibido; 3) "tiempo", sería la cantidad de minutos semanales dedicados al entrenamiento; 4) "tipo", se refiere a la modalidad de ejercicios específicos que se están prescribiendo, por ejemplo, ejercicios isométricos; 5) "volumen", indica las series y repeticiones; y 6) "progresión", consiste en la manipulación explícita de las variables del FITT-V para lograr nuevas adaptaciones.

Es indiscutible la importancia que tiene el diseño sistemático del programa de ejercicio terapéutico, sin embargo, hay que tener en cuenta que la efectividad del ejercicio prescrito depende de muchos más factores, sobre todo cuando hablamos de trastornos que presenten alta cronicidad. Factores cognitivos y afectivos-motivacionales tienen gran relevancia en las 
expectativas, autoeficacia, la motivación al cambio, el compromiso y la adhesión al ejercicio terapéutico. Contamos con evidencia científica que demuestra que la utilización de métodos dirigidos a modificar el comportamiento, mejoran los resultados en términos de adhesión al ejercicio y disminución de la discapacidad (Bunzli, Gillham, \& Esterman, 2011; Button, Roos, Spasić, Adamson, \& van Deursen, 2015; Marley et al., 2017; Meade, Bearne, Sweeney, Alageel, \& Godfrey, 2019; Moyer, Ikert, Long, \& Marsh, 2017; Willett et al., 2019).

El concepto FITT-VP aplicado al ejercicio terapéutico debe de evolucionar hacia una perspectiva bioconductual. Una propuesta que podría ser útil es trabajar bajo el concepto FITTV-EPA (F, frecuencia; I, intensidad; T, tiempo; $T$, tipo de ejercicio; $V$, volumen; E, educación; P, planteamiento de objetivos; A, análisis bioconductual). El acrónimo EPA engloba la estrategia de educación terapéutica basado en el paciente para favorecer los cambios del comportamiento y que los pacientes se adapten y acepten mejor los programas de ejercicio terapéutico. Esta estrategia incluiría un análisis previo de los factores cognitivos, expectativas y factores afectivosmotivacionales que pueden actuar como interferencias potenciales en la práctica del ejercicio terapéutico (análisis bioconductual), un planteamiento de objetivos razonado y estructurado, y finalmente un proceso educativo sobre los aspectos positivos del ejercicio y sobre las posibles percepciones asociadas a la práctica del ejercicio.

Los fisioterapeutas deben de dirigir parte de sus esfuerzos a educar al paciente previo o a la vez de que se diseña el programa de prescripción de ejercicio. La educación terapéutica es un tratamiento cada vez más estudiado y utilizado en la práctica de la fisioterapia la cual, se define como una modalidad terapéutica que de forma explícita conlleva a un sistema de comunicación e interacción dinámica no direccional con el paciente, basada en un paradigma bioconductual que incluye actividades educacionales o formativas que promocionan el aprendizaje y adquisición de habilidades adaptativas para mejorar la autogestión y conocimientos que faciliten los cambios en creencias, actitudes y comportamientos asociados a la pérdida de la función. La evidencia científica actual sugiere que diversas estrategias incluidas dentro de la educación terapéutica influyen sobre los resultados del ejercicio terapéutico (Button et al., 2015; Jones, Valenzuela, Booth, Taylor, \& Barry, 2017; Jönsson et al., 2018) y debería normalizarse su uso junto a la prescripción de ejercicio terapéutico.

La fisioterapia como profesión debe de posicionarse como un referente en el sistema del movimiento humano a nivel clínico, para esto es necesario que se incremente el control y conocimiento avanzado en competencias de diagnóstico basado en la función, en prescripción de ejercicio y educación terapéutica; este es el factor clave para lograr un mayor reconocimiento social y un impacto real sobre las futuras demandas sanitarias a las que se enfrenta el mundo. Considero que el punto central de unión de la profesión a nivel científico, académico y clínico debe de estar en torno a todos los temas derivados del conocimiento práctico del sistema del movimiento en los entornos clínico. Las universidades deben de pasar a la acción de forma inmediata. El cambio de los programas de estudios no puede esperar más. Los científicos debemos volcar nuestros esfuerzos de investigación a incrementar el cuerpo de evidencia científica hacia el ejercicio terapéutico y hacia las modalidades de diagnóstico funcional.

Esta revista quiere colaborar de forma activa con este propósito, siendo un medio para divulgar ciencia sobre el sistema del movimiento humano. Esperemos que las investigaciones publicadas en este número sirvan para incrementar el conocimiento y despertar el espíritu crítico.

\section{REFERENCIAS}

American Physical Therapy Association. Physical Therapist Practice and The Human Movement System. APTA. 2015.

Bunzli S, Gillham D, Esterman A. Physiotherapy-provided operant conditioning in the management of low back pain disability: A systematic review. Physiother Res Int. Physiother Res Int; 2011;16(1):4-19 DOI: http://dx.doi.org/10.1002/pri.465.

Button K, Roos PE, Spasić I, Adamson P, van Deursen RWM. The clinical effectiveness of self-care interventions with an exercise component to manage knee conditions: A systematic review. Knee; 2015;22:360-71 DOI: http://dx.doi.org/10.1016/ j.knee. 2015.05.003.

Caspersen CJ, Powell KE, Christenson GM. Physical activity, exercise, and physical fitness: definitions and distinctions for 
health-related research. Public Health Rep. 1985;100(2):126-31 DOI: http://dx.doi.org/10.2307/20056429.

Coffin-Zadai CA. Disabling Our Diagnostic Dilemmas. Phys Ther. Oxford University Press (OUP); 2007;87(6):641-53 DOI: http://dx.doi.org/10.2522/ptj.20060236.

Fernández-López JA, Fernández-Fidalgo M, Cieza A. Los conceptos de calidad de vida, salud y bienestar analizados desde la perspectiva de la clasificación internacional del funcionamiento (CIF). Rev Esp Salud Publica. Rev Esp Salud Publica; 2010;84(2):169-84 DOI: http://dx.doi.org/10.1590/s1135-572720 10000200005.

Garber CE, Blissmer B, Deschenes MR, Franklin BA, Lamonte MJ, Lee I-M, Nieman DC, Swain DP, American College of Sports Medicine. American College of Sports Medicine position stand. Quantity and quality of exercise for developing and maintaining cardiorespiratory, musculoskeletal, and neuromotor fitness in apparently healthy adults: guidance for prescribing exercise. Med Sci Sports Exerc. 2011;43(7):1334-59 DOI: http://dx.doi.org/10. 1249/MSS.0b013e318213fefb.

Hansson N, Ottosson A. Nobel Prize for Physical Therapy? Rise, Fall, and Revival of Medico-Mechanical Institutes. Phys Ther. Oxford University Press (OUP); 2015;95(8):1184-94 DOI: http://dx.doi.org/10.2522/ptj.20140284.

Jones MD, Valenzuela T, Booth J, Taylor JL, Barry BK. Explicit Education About Exercise-Induced Hypoalgesia Influences Pain Responses to Acute Exercise in Healthy Adults: A Randomized Controlled Trial. J Pain. Churchill Livingstone Inc.; 2017;18(11):1409-16 DOI: http://dx.doi.org/10.1016/j.jpain.2017. 07.006 .

Jönsson T, Ekvall Hansson E, Thorstensson CA, Eek F, Bergman P, Dahlberg LE. The effect of education and supervised exercise on physical activity, pain, quality of life and self-efficacy an intervention study with a reference group. BMC Musculoskelet Disord. BioMed Central Ltd.; 2018;19(1):198 DOI: http://dx.doi.org/10.1186/s12891-018-2098-3.

Kottke FJ, Stillwell GK LJ. Krusen's Handbook of Physical Medicine and Rehabilitation. 3rd ed. Philadelphia: WB Saunders Co 1982.

Marley J, Tully MA, Porter-Armstrong A, Bunting B, O'Hanlon J, Atkins L, Howes S, McDonough SM. The effectiveness of interventions aimed at increasing physical activity in adults with persistent musculoskeletal pain: A systematic review and meta-analysis. BMC Musculoskeletal Disorders; 2017;22;18(1):482. DOI: http://dx.doi.org/10.1186/s12891-0171836-2.
Meade LB, Bearne LM, Sweeney LH, Alageel SH, Godfrey EL. Behaviour change techniques associated with adherence to prescribed exercise in patients with persistent musculoskeletal pain: Systematic review. Br J Health Psychol. John Wiley and Sons Ltd.; 2019;24(1):10-30 DOI: http://dx.doi.org/10.1111/bjhp.12324.

Moyer R, Ikert K, Long K, Marsh J. The Value of Preoperative Exercise and Education for Patients Undergoing Total Hip and Knee Arthroplasty: A Systematic Review and Meta-Analysis. JBJS Rev. NLM (Medline); 2017;5(12):e2 DOI: http://dx.doi.org/ 10.2106/JBJS.RVW.17.00015.

Nyland JA. Redirecting the thrust to put "therapeutic" back into therapeutic exercise. Vol. 45, Journal of Orthopaedic and Sports Physical Therapy. Movement Science Media; 2015. p. 148-50 DOI: http://dx.doi.org/10.2519/jospt.2015.0103.

Sahrmann SA. The Human Movement System: Our Professional Identity. Phys Ther. Oxford University Press (OUP); 2014;94(7):1034-42 DOI: http://dx.doi.org/10.2522/ptj.20130319.

Shaik AR, Maniyat Shemjaz A. Medical History The rise of physical therapy: A history in footsteps. Arch Med Heal Sci. 2014;2(2):257-60 DOI: http://dx.doi.org/10.4103/2321-4848. 144367

Thivel D, Tremblay A, Genin PM, Panahi S, Rivière D, Duclos M. Physical Activity, Inactivity, and Sedentary Behaviors: Definitions and Implications in Occupational Health. Front Public Heal. Frontiers Media SA; 2018;6 DOI: http://dx.doi.org/10.3389/fpubh.2018.00288.

La Touche R. Rehabilitación de tercera generación. NeuroRehab News. 2017;2(1):e0019.

Voight ML, Hoogenboom BJ. What is the movement system and why is it important? Int J Sports Phys Ther. The Sports Physical Therapy Section of the American Physical Therapy Association; 2017;12(1):1-2.

Willett M, Duda J, Fenton S, Gautrey C, Greig C, Rushton A. Effectiveness of behaviour change techniques in physiotherapy interventions to promote physical activity adherence in lower limb osteoarthritis patients: A systematic review. PLoS One. Public Library of Science; 2019;14(7) DOI: http://dx.doi.org/10.1371/ journal.pone.0219482.

Zaleski AL, Taylor BA, Panza GA, Wu Y, Pescatello LS, Thompson PD, Fernandez AB. Coming of Age: Considerations in the Prescription of Exercise for Older Adults. Vol. 12, Methodist DeBakey cardiovascular journal. Methodist DeBakey Heart \& Vascular Center; 2016;12(2);98-104. DOI: http://dx.doi.org/ 10.14797/mdcj-12-2-98 